\title{
Distinguishing unentangled states with an unentangled measurement
}

\author{
William K. Wootters \\ Department of Physics, Williams College, Williamstown, MA 01267
}

\begin{abstract}
In a 1991 paper, Asher Peres and the author theoretically analyzed a set of unentangled bipartite quantum states that could apparently be distinguished better by a global measurement than by any sequence of local measurements on the individual subsystems. The present paper returns to the same example, and shows that the best result so far achieved can alternatively be attained by a measurement that, while still global, is "unentangled" in the sense that the operator associated with each measurement outcome is a tensor product.
\end{abstract}




\section{Introduction}

In the fall of 1989, I had the good fortune to be at the Santa Fe Institute at the same time as Asher Peres, and we quickly began working together on the following problem.

A quantum system consists of two spatially separated components. The system is known to be in one of several possible pure states, each of which is a product state, and one wants to perform a measurement that will provide as much information as possible about the identity of the system's state. One can consider two kinds of measurement: (i) a sequence of local measurements on the individual subsystems, each measurement possibly depending on the outcomes of earlier measurements; (ii) a single global measurement on the system as a whole. Question: In order to gain as much information as possible, is it sometimes necessary to use a global measurement, or can one always do just as well with a cleverly chosen sequence of local measurements?

The states that one is trying to distinguish are all product states; so one might have expected that local measurements would be sufficient. Indeed, this was my intuition at the time. Fortunately it contrasted nicely with Asher's intuition, and the contrast helped fuel a stimulating collaboration. At first we considered a few examples in which one was trying to distinguish just two states; in these cases we found no advantage to a global measurement. (A few years later, Ban et al. [1], following work by Brody and Meister [2], showed that for distinguishing between just two states $\left|\psi_{1}\right\rangle \otimes\left|\psi_{1}\right\rangle$ and $\left|\psi_{2}\right\rangle \otimes\left|\psi_{2}\right\rangle$, the optimal amount of information can always be obtained by a sequence of local measurements. Recently a similar conclusion was reached, for an arbitrary number of copies, by Acin et al. 3.).) Finally, though, we hit upon a three-state example for which a global measurement was distinctly better than any adaptive local strategy that we could devise [4. Though the superiority of global measurements in this particular example has still not been proved conclusively, other studies, based on other examples, have decisively confirmed that Asher's intuition about global measurements was correct [5, 6, 7].

In the present paper I return to the same three-state example that we originally considered - the "double-trine" ensemble - in order to explore a further 
distinction among measurements. In 1989 Asher convinced me (and I remain convinced) that no sequence of local measurements can match the best global measurement in discriminating among the three double-trine states. But among all complete, global measurements, it is interesting to consider two mutually exclusive and exhaustive classes: (i) those whose outcomes are associated with product states, and (ii) those for which at least one outcome is associated with an entangled state. I will call the former measurements "unentangled" and the latter "entangled." 1 One might have thought that any measurement whose outcomes are associated with product states could be carried out as a sequence of local measurements, but this is not the case [5]. That is, the class of unentangled measurements is strictly larger than the class of measurements that can be realized locally, even with classical communication. Still, unentangled measurements are quite special. For example, like local adaptive measurements, they do not create entanglement where none existed previously. And as a class they are more tractable mathematically than sequences of local measurements. So it is interesting to ask how effective such measurements can be, and to what extent they are limited relative to the much larger class of entangled measurements.

For the double-trine ensemble, the best measurement that Asher and I found was not merely a global measurement but also an entangled measurement. So our result left open the question whether an unentangled measurement could do just as well. The main purpose of the present paper is to show that an unentangled measurement can in fact do just as well, as we will see in Section 3.

Though this paper focuses on a specific example, it raises a more general question. We know by now several examples of ensembles of product states for which the optimal discrimination cannot be achieved by a sequence of local measurements (see, for example, Refs. [5, 6, 7]). However, I have not found in the literature any example for which it is known that the optimal discrimination cannot be achieved by an unentangled measurement. The case that Asher and I considered might have seemed a good candidate for such an example, since the best measurement that we found is entangled. But the result of the present paper rules out this case. One wonders, then,

\footnotetext{
${ }^{1}$ Some authors, including the present author, have referred to measurements of the former class as "separable measurements," since they can be implemented by separable superoperators [5, 6, 7]. But other authors have reserved the term "separable" for sequences of local measurements (see, for example, Refs. [8, 9, 10]). In this paper I am using the term "unentangled measurement" in order to avoid confusion.
} 
whether every ensemble of product states can be optimally distinguished by an unentangled measurement. Though this may seem unlikely, it has apparently not been shown to be false.

Asher returned to Israel before we had finished working on the problem, so we continued our collaboration by email. I still have hard copies of most if not all of the notes that I received from him during those months; most of these advance the research itself but some address other matters. In one of these emails, Asher responds to a very early draft of our paper that I had sent him, in which I had used the word "considerably," and at one point, also the word "mind," as in "the particles' correlation is only in the mind of the observer." He politely noted that he would prefer not to use the word "mind" in this context-he said that everyone is free to interpret the formalism according to one's cultural background-and that in general he preferred to avoid the words "extremely" and "considerably," a practice he learned from Larry Schulman and later found supported in The Elements of Style by Strunk and White. I confess that I did not learn this lesson and have allowed "considerably" to slip into many papers since then. But in honor of Asher, and for the sake of good style, I will resist the temptation to use either "considerably" or "extremely" in the rest of this paper. There should be no need for "mind" either.

\section{The double-trine ensemble}

The ensemble considered in Ref. 4] consists of three pure product states of a pair of qubits, each state having a priori probability $1 / 3$. Each qubit is in one of three states equally spaced on a great circle of the Bloch sphere, and the two qubits are both in the same state. We represent the three single-qubit states - called the "trine" states - as

$$
\left|\psi_{0}\right\rangle=\left(\begin{array}{l}
1 \\
0
\end{array}\right) \quad\left|\psi_{1}\right\rangle=\left(\begin{array}{c}
-1 / 2 \\
-\sqrt{3} / 2
\end{array}\right) \quad\left|\psi_{2}\right\rangle=\left(\begin{array}{c}
-1 / 2 \\
\sqrt{3} / 2
\end{array}\right)
$$

Here the overall phases of the vectors have been chosen so that $\left\langle\psi_{i} \mid \psi_{j}\right\rangle$ has the same value, $-1 / 2$, for all $i \neq j$. (This choice makes some later equations simpler.)

The three possible states of the pair of qubits are

$$
\left|a_{j}\right\rangle=\left|\psi_{j}\right\rangle \otimes\left|\psi_{j}\right\rangle, \quad j=0,1,2 \text {. }
$$


(I will use Latin letters for two-qubit states and Greek letters for single-qubit states.) We imagine that we are presented with a single copy of such a pair of qubits; our goal is to perform a measurement on the pair that distinguishes the three states as well as possible.

As our measure of success, we use the mutual information between the outcome of our measurement and the index $j$ that identifies the state $(j=$ $0,1,2)$. Let the outcome of the measurement be labeled by $k=1, \ldots, M$, where the number of outcomes, $M$, need not be the same as the number of states in our ensemble. The mutual information $I$ is

$$
I=H(\text { state })+H(\text { outcome })-H(\text { state and outcome }),
$$

where $H$ is the Shannon entropy of the indicated probability distribution. Let $p_{k}$ be the probability of outcome $k$ (averaged over the possible states of the system), and let $p_{j k}$ be the probability that the state is $\left|a_{j}\right\rangle$ and the measurement outcome is $k$. Then for our three-state example we can write the mutual information as

$$
I=\log 3-\sum_{k=1}^{M} p_{k} \log p_{k}+\sum_{j=0}^{2} \sum_{k=1}^{M} p_{j k} \log p_{j k},
$$

in which we take the base of the logarithms to be 2. For our purposes, a somewhat more convenient form is

$$
I=-\sum_{k=1}^{M} p_{k} \log p_{k}+(1 / 3) \sum_{j=0}^{2} \sum_{k=1}^{M} p_{k \mid j} \log p_{k \mid j}
$$

where $p_{k \mid j}$ is the probability of outcome $k$ given that the state is $\left|a_{j}\right\rangle$. In this form, it reads as the average amount of information one gains about the outcome of the measurement upon learning the identity of the state, but the mutual information can also be interpreted as the average amount of information that one gains about the state upon seeing the outcome of the measurement. Note that with three equally likely states in the ensemble, the maximum conceivable value of the mutual information is $\log 3=1.585$ bits, the value we would get if the measurement outcome were perfectly correlated to the system's state. We will certainly not achieve this value, since our three states are not mutually orthogonal and can therefore not be distinguished perfectly. 
In Ref. 4] a number of different measurement strategies for this problem were considered, the best being an orthogonal joint measurement on the pair of qubits. The construction of this measurement is motivated by the observation that the three states $\left|a_{0}\right\rangle,\left|a_{1}\right\rangle$, and $\left|a_{2}\right\rangle$ can be regarded as unit vectors in a real vector space, the angle between each pair being $75.5^{\circ}$. (This angle is the inverse cosine of $1 / 4$, which is the inner product between any two of these vectors.) There is a unique triple of orthogonal vectors in this real vector space that symmetrically straddles the vectors $\left|a_{0}\right\rangle,\left|a_{1}\right\rangle$, and $\left|a_{2}\right\rangle$ and approximates them as closely as possible. One finds that the three orthogonal vectors are

$$
\left|A_{j}\right\rangle=\frac{1}{3 \sqrt{3}}\left[(4+\sqrt{2})\left|a_{j}\right\rangle-(2-\sqrt{2})\left(\left|a_{j+1}\right\rangle+\left|a_{j+2}\right\rangle\right)\right], \quad j=0,1,2,
$$

where the addition in the subscripts is mod 3. To complete the measurement on the pair of qubits, we need a fourth state orthogonal to each of the states $\left|A_{j}\right\rangle$. This is the singlet state

$$
|S\rangle=\frac{1}{\sqrt{2}}(|01\rangle-|10\rangle),
$$

where $|0\rangle$ and $|1\rangle$ are our standard basis states for a single qubit. One can use the relation $\left\langle a_{i} \mid a_{j}\right\rangle=(1 / 4)+(3 / 4) \delta_{i j}$ to verify that the states $\left|A_{j}\right\rangle$ are indeed mutually orthogonal. That they are also all orthogonal to the singlet state follows from the construction of $\left|a_{j}\right\rangle$ as a repeated single-qubit state, which places it in the triplet subspace. This latter orthogonality implies that for the given ensemble, the outcome $|S\rangle$ will never happen. So for our purpose this measurement has, in effect, only three possible outcomes.

One can show that the three states $\left|A_{j}\right\rangle$ are all entangled, as is the singlet state $|S\rangle$. So the measurement defined by these states is an entangled measurement.

How large a value of the mutual information does this measurement provide? To answer this question we need to compute the probability of the outcome $\left|A_{k}\right\rangle$ given the initial state $\left|a_{j}\right\rangle$. For the case $k=j$, we have

$$
p_{j \mid j}=\left|\left\langle a_{j} \mid A_{j}\right\rangle\right|^{2}=\left\{\frac{1}{3 \sqrt{3}}[(4+\sqrt{2})-(1 / 2)(2-\sqrt{2})]\right\}^{2}=\frac{1}{2}+\frac{\sqrt{2}}{3},
$$

which is about 0.971. (In the real-vector-space picture described above, the angle between each vector $\left|a_{j}\right\rangle$ and its corresponding $\left|A_{j}\right\rangle$ is $\cos ^{-1}(\sqrt{0.971}) \approx$ 
$10^{\circ}$.) The other two outcomes share the remaining probability equally; so for $k \neq j$ we have

$$
p_{k \mid j}=\frac{1}{2}\left[1-\left(\frac{1}{2}+\frac{\sqrt{2}}{3}\right)\right]=\frac{1}{4}-\frac{1}{3 \sqrt{2}},
$$

which is about 0.014 . The three measurement outcomes have equal a priori probability; so according to Eq. (5), the mutual information is

$$
I=\log 3+(0.971) \log (0.971)+2(0.014) \log (0.014)=1.369 \text { bits }
$$

which is not much less than the upper bound $\log 3=1.585$ bits. Because of the symmetry of the problem and the proximity of each outcome vector $\left|A_{j}\right\rangle$ to the corresponding state vector $\left|a_{j}\right\rangle$, it is plausible that the measurement we have just described is optimal. Numerical work similarly suggests that it is optimal [11, but this conjecture has apparently never been proved, and I have no proof of it to offer here. (Refs. [12, 13, 14 prove that it is optimal in different senses, that is, with measures of success other than the mutual information.)

The other measurement strategies considered in Ref. [4] are all sequences of local measurements, the most sophisticated being a back-and-forth sequence of successively stronger measurements, alternating between the two qubits. But the highest value of mutual information obtained in this way was $1.26205 \pm 0.00037$ (the uncertainty was computed from a Monte Carlo simulation), and it seems unlikely that any such sequence will match the value 1.369 obtained from a global measurement.

As far as I know, this last conjecture has also never been proved. However, the claim that global measurements can be better, even when the states in question are product states, has been demonstrated decisively in other contexts, as I have noted in the Introduction. Bennett et al. have given an example of a set of orthogonal product states that cannot be distinguished by any sequence of local measurements, even though, being orthogonal, they certainly can be distinguished by a global measurement [5]. Similar examples have been given as part of the study of "unextendible product bases" [6. 17, and efficient proofs of this sort of indistinguishability have been devised [15, 16]. There is also an extensive literature on the problem of ascertaining a quantum state, or some parameter of a quantum state, when many instances of the state are provided; many of these papers distinguish between 
local and global measurements and ask whether the latter allows a more precise estimate (see, for example, Refs. 17, 8, 9, 3, 10] and references cited therein). One finds that the existence of an advantage conferred by global measurements depends on the precise question being asked.

In the following section I continue analyzing the double-trine ensemble, asking in particular how well the three states can be distinguished by an unentangled measurement.

\section{An unentangled measurement}

Consider a single qubit, known to be in one of the three trine states $\left|\psi_{j}\right\rangle$, $j=0,1,2$, with equal a priori probabilities. It has been shown ${ }^{2}$ that the optimal mutual information for this case can be obtained by a three-outcome, non-orthogonal measurement represented by the following positive-operatorvalued measure (POVM):

$$
\Pi_{k}=\frac{2}{3}\left|\psi_{k}^{\perp}\right\rangle\left\langle\psi_{k}^{\perp}\right|, \quad k=0,1,2 .
$$

Here the operators $\Pi_{k}$ are the positive operators representing the outcomes of the measurement - their sum is the $2 \times 2$ identity - and $\left|\psi_{k}^{\perp}\right\rangle$ is the qubit state orthogonal to $\left|\psi_{k}\right\rangle$. The probability of outcome $k$ if the qubit is in the state $\left|\psi_{j}\right\rangle$ is

$$
p_{k \mid j}=\left\langle\psi_{j}\left|\Pi_{k}\right| \psi_{j}\right\rangle=\left\{\begin{array}{l}
0, \text { if } k=j \\
1 / 2, \text { if } k=j+1 \\
1 / 2, \text { if } k=j+2
\end{array}\right.
$$

where the addition is mod 3. Thus this measurement has the effect of ruling out one of the three possible states and leaving the other two equally likely. The mutual information is $I=\log 3-\log 2=0.585$ bits, which is strictly larger than any value that can be obtained with an orthogonal measurement.

Let us try to use the idea behind this measurement to guide us in designing an unentangled measurement for the double-trine ensemble. As indicated in the Introduction, by "unentangled" I mean that each of the operators of the POVM will be a tensor product. Now, initially we have three possible states of the pair of qubits. Ideally, we would like to rule out two of these

\footnotetext{
${ }^{2}$ This three-outcome POVM was proposed for this ensemble by Holevo 18, who showed that it provided more information than any orthogonal measurement. That this POVM is in fact optimal was proved by Sasaki et al. [19].
} 
states by our measurement. We have just seen an example of a single-qubit measurement that rules out one of the three. Since we now have two qubits to work with, we can try to use them to rule out two states, thereby leaving us with only the correct state. (We know in advance that this will not in fact be possible, since the three states of our ensemble are not orthogonal, but let us see how well we can do.) As our first guess towards a good POVM, consider the following set:

$$
\left\{\Pi_{0} \otimes \Pi_{1}, \Pi_{1} \otimes \Pi_{0}, \Pi_{1} \otimes \Pi_{2}, \Pi_{2} \otimes \Pi_{1}, \Pi_{2} \otimes \Pi_{0}, \Pi_{0} \otimes \Pi_{2}\right\},
$$

where each of the operators $\Pi_{j}$ is chosen from the single-qubit POVM given in Eq. (11). The reader may already have noticed that this set does not actually constitute a POVM, because its elements do not add up to the $4 \times 4$ identity operator. Ignoring for now this annoying fact, we note that any of the six operators listed here would be ideal as an element of a POVM, since each rules out two of the states of our ensemble. For example, $\left\langle a_{j}\left|\Pi_{0} \otimes \Pi_{1}\right| a_{j}\right\rangle$ is nonzero only if $j=2$.

Can we modify slightly the set given in Eq. (13) so as to create a legitimate POVM whose effect approximates the ideal? One approach would be to include three additional elements: $\Pi_{0} \otimes \Pi_{0}, \Pi_{1} \otimes \Pi_{1}$, and $\Pi_{2} \otimes \Pi_{2}$. Then one is in effect performing on each of the qubits the POVM given in Eq. (11), and this is a perfectly legitimate measurement on the pair. It turns out, though, that this is not a very effective strategy: the mutual information is $\log 3-1 / 2=1.085$ bits, which is not nearly as large as for the entangled measurement considered in Section 2. ${ }^{3}$ (The reason for the poor discrimination is that these last three elements, each of which rules out only one of the three states, have a combined probability equal to the combined probability of the six "good" POVM elements listed in Eq. (13).)

A better strategy is to construct a POVM with only six elements by modifying slightly each of the operators in Eq. (13). Rather than insisting that each POVM element definitively rule out two of the states $\left|\psi_{j}\right\rangle$, we look for POVM elements that will make two of the states very unlikely. To this

\footnotetext{
${ }^{3}$ Though this particular strategy is not mentioned in Ref. [4, on looking back over the email correspondence, I see that Asher did consider this possibility. In a note dated 5 Nov 1989, he observed that even though this method does not require classical communication between the two parties, the amount of information it provides, $1.085 \mathrm{bits}$, is larger than what can be obtained with a pair of local orthogonal measurements connected by classical communication.
} 
end, we define six single-qubit states as follows:

$$
\left|\phi_{j}^{+}\right\rangle=R\left|\psi_{j}\right\rangle, \quad\left|\phi_{j}^{-}\right\rangle=R^{-1}\left|\psi_{j}\right\rangle, \quad j=0,1,2 .
$$

Here the unitary matrix $R$ is given by

$$
R=\left(\begin{array}{cc}
\cos (\theta / 2) & -\sin (\theta / 2) \\
\sin (\theta / 2) & \cos (\theta / 2)
\end{array}\right)
$$

where the angle $\theta$ is yet to be determined. In the $x-z$ plane of the Bloch sphere, the states $\left|\phi_{j}^{+}\right\rangle$and $\left|\phi_{j}^{-}\right\rangle$each make an angle $\theta$ with $\left|\psi_{j}\right\rangle$ and lie on opposite sides of it.

We now consider the following six two-qubit states:

$$
\left|B_{j}\right\rangle=\left|\phi_{j}^{+}\right\rangle \otimes\left|\phi_{j}^{-}\right\rangle, \quad\left|C_{j}\right\rangle=\left|\phi_{j}^{-}\right\rangle \otimes\left|\phi_{j}^{+}\right\rangle, \quad j=0,1,2 .
$$

Our new candidate for a good POVM consists of the operators

$$
E_{j}=\alpha\left|B_{j}\right\rangle\left\langle B_{j}\left|, \quad F_{j}=\alpha\right| C_{j}\right\rangle\left\langle C_{j}\right|, \quad j=0,1,2,
$$

where $\alpha$ is a constant whose value is to be determined. We want to choose $\theta$ and $\alpha$ so that the set $\mathcal{E}=\left\{E_{1}, E_{2}, E_{3}, F_{1}, F_{2}, F_{3}\right\}$ constitutes a POVM.

Notice that if $\alpha=4 / 9$ and $\theta=60^{\circ}$, the set $\mathcal{E}$ is identical to the set given in Eq. (13). In that case, for example, $\left|\phi_{0}^{+}\right\rangle$equals $\left|\psi_{2}^{\perp}\right\rangle$ and $\left|\phi_{0}^{-}\right\rangle$equals $\left|\psi_{1}^{\perp}\right\rangle$, so that $E_{0}$ becomes proportional to $\Pi_{2} \otimes \Pi_{1}$. But this choice does not give us a POVM. For arbitrary $\alpha$ and $\theta$, one can work out the sum of the elements of $\mathcal{E}$; the result is

$$
\sum_{j=0}^{2}\left(E_{j}+F_{j}\right)=\alpha\left(\frac{3}{2}\right)\left[I+\frac{1}{2} \cos (2 \theta)\left(\sigma_{x} \otimes \sigma_{x}+\sigma_{z} \otimes \sigma_{z}\right)\right],
$$

where $\sigma_{x}$ and $\sigma_{z}$ are Pauli matrices. This sum is equal to the identity only if $\alpha=2 / 3$ and $\cos (2 \theta)=0$. (That $\alpha$ must equal $2 / 3$ can in fact be derived more easily just by insisting that the traces of the elements of $\mathcal{E}$ add up to the trace of the identity.) We thus obtain a POVM by choosing $\theta$ to be either $45^{\circ}$ or $135^{\circ}$. The choice $\theta=45^{\circ}$ is closer to our ideal of $60^{\circ}$, and indeed one finds that this choice provides a significantly larger mutual information.

With this choice $-\alpha=2 / 3$ and $\theta=\pi / 4$ - let us now compute the mutual information. Because of the symmetry, it is sufficient to find the probability 
of, say, the outcome $E_{0}$ when the state is $\left|a_{0}\right\rangle$; all other probabilities can be computed from this one. This probability is

$$
\text { (probability of } \left.E_{0} \text { given }\left|a_{0}\right\rangle\right)=\left\langle a_{0}\left|E_{0}\right| a_{0}\right\rangle=\frac{2}{3}\left|\left\langle\psi_{0} \mid \phi_{0}^{+}\right\rangle\right|^{2}\left|\left\langle\psi_{0} \mid \phi_{0}^{-}\right\rangle\right|^{2} \text {. }
$$

Now, the state $\left|\phi_{0}^{+}\right\rangle$is $45^{\circ}$ away from $\left|\psi_{0}\right\rangle$ on the Bloch sphere, and so is $\left|\phi_{0}^{-}\right\rangle$. So $\left|\left\langle\psi_{0} \mid \phi_{0}^{+}\right\rangle\right|^{2}=\left|\left\langle\psi_{0} \mid \phi_{0}^{-}\right\rangle\right|^{2}=\cos ^{2}(\pi / 8)$. The above probability therefore becomes

$$
\text { (probability of } \left.E_{0} \text { given }\left|a_{0}\right\rangle\right)=\frac{2}{3} \cos ^{4}(\pi / 8)=\frac{1}{4}+\frac{\sqrt{2}}{6}=0.486 .
$$

The probability of $F_{0}$ given $\left|a_{0}\right\rangle$ must have this same value, and the remaining probability must be split equally among the four remaining outcomes. Thus, if the initial state is $\left|a_{0}\right\rangle$, the probabilities of the six outcomes are

$$
\frac{1}{4}+\frac{\sqrt{2}}{6}, \frac{1}{4}+\frac{\sqrt{2}}{6}, \frac{1}{8}-\frac{\sqrt{2}}{12}, \frac{1}{8}-\frac{\sqrt{2}}{12}, \frac{1}{8}-\frac{\sqrt{2}}{12}, \frac{1}{8}-\frac{\sqrt{2}}{12} .
$$

Moreover, because of the symmetry of the problem we get the same set of values for each of the other two possible initial states $\left|a_{1}\right\rangle$ and $\left|a_{2}\right\rangle$. The six measurement outcomes are all equally likely a priori; so the mutual information is

$$
I=\log 6+2\left(\frac{1}{4}+\frac{\sqrt{2}}{6}\right) \log \left(\frac{1}{4}+\frac{\sqrt{2}}{6}\right)+4\left(\frac{1}{8}-\frac{\sqrt{2}}{12}\right) \log \left(\frac{1}{8}-\frac{\sqrt{2}}{12}\right)
$$

which comes out to be 1.369 bits. This is exactly what we got with the entangled measurement of Section 2. Indeed, by comparing Eq. (20) with Eq. (8)), one can already see that the mutual information for the two cases will be the same. (Each probability for the six-outcome measurement is one-half of the corresponding value for the three-outcome entangled measurement, but the a priori probabilities are also half as great, and this overall factor of one-half has no effect on the mutual information.)

We have thus found an alternative measurement that is just as good as our best entangled measurement (at least, the best that is known so far). And this alternative measurement is unentangled. The exact equivalence is perhaps a little surprising, since our construction of the unentangled measurement is quite different from that of the entangled measurement and is 
motivated by a different heuristic strategy. In fact, one might even wonder why the unentangled measurement could not have been a little better than the entangled one.

The following section shows that there is, after all, a connection between the two cases, and that the equivalence between these two measurements, regarded as strategies for discriminating the states of the double-trine ensemble, is not as surprising as it might at first seem.

\section{Assimilating the singlet state}

Recall that the entangled measurement discussed in Section 2 is defined by the orthonormal basis $\left\{\left|A_{0}\right\rangle,\left|A_{1}\right\rangle,\left|A_{2}\right\rangle,|S\rangle\right\}$, the last element of which is the singlet state. The unentangled measurement of Section 3 is defined by six non-orthogonal states $\left\{\left|B_{0}\right\rangle,\left|B_{1}\right\rangle,\left|B_{2}\right\rangle,\left|C_{0}\right\rangle,\left|C_{1}\right\rangle,\left|C_{2}\right\rangle\right\}$. It is useful to write a few of these states out explicitly in the standard basis $\{|00\rangle,|01\rangle,|10\rangle,|11\rangle\}$ :

$$
\left|A_{0}\right\rangle=\frac{1}{\sqrt{6}}\left(\begin{array}{c}
1+\sqrt{2} \\
0 \\
0 \\
1-\sqrt{2}
\end{array}\right) \quad\left|B_{0}\right\rangle=\frac{1}{2 \sqrt{2}}\left(\begin{array}{c}
1+\sqrt{2} \\
-1 \\
1 \\
1-\sqrt{2}
\end{array}\right) \quad\left|C_{0}\right\rangle=\frac{1}{2 \sqrt{2}}\left(\begin{array}{c}
1+\sqrt{2} \\
1 \\
-1 \\
1-\sqrt{2}
\end{array}\right)
$$

In terms of the singlet state $|S\rangle=(1 / \sqrt{2})(|01\rangle-|10\rangle)$, we can write $\left|B_{0}\right\rangle$ and $\left|C_{0}\right\rangle$ as

$$
\left|B_{0}\right\rangle=\frac{\sqrt{3}}{2}\left|A_{0}\right\rangle-\frac{1}{2}|S\rangle, \quad\left|C_{0}\right\rangle=\frac{\sqrt{3}}{2}\left|A_{0}\right\rangle+\frac{1}{2}|S\rangle .
$$

A similar relation holds for the other values of the index $j$. To see this, note that the single-qubit unitary operator

$$
u=\left(\begin{array}{cc}
-1 / 2 & \sqrt{3} / 2 \\
-\sqrt{3} / 2 & -1 / 2
\end{array}\right)
$$

maps our three initial single-qubit states to each other cyclically; that is, for each $j=0,1,2$, we have $u\left|\psi_{j}\right\rangle=\left|\psi_{j+1}\right\rangle$, where the addition is $\bmod 3$ as usual. It follows that the tensor product operator $U=u \otimes u$ generates similar cycles among our two-qubit states:

$$
U\left|A_{j}\right\rangle=\left|A_{j+1}\right\rangle, \quad U\left|B_{j}\right\rangle=\left|B_{j+1}\right\rangle, \quad U\left|C_{j}\right\rangle=\left|C_{j+1}\right\rangle, \quad j=0,1,2 .
$$


Moreover, $U$ leaves the singlet state $|S\rangle$ invariant; so applying $U$ to each term in Eq. (24), we get

$$
\left|B_{j}\right\rangle=\frac{\sqrt{3}}{2}\left|A_{j}\right\rangle-\frac{1}{2}|S\rangle, \quad\left|C_{j}\right\rangle=\frac{\sqrt{3}}{2}\left|A_{j}\right\rangle+\frac{1}{2}|S\rangle, \quad j=0,1,2 .
$$

Thus the states that define our unentangled measurement are quite closely related to the states of our entangled measurement: the separable states $\left|B_{j}\right\rangle$ and $\left|C_{j}\right\rangle$ are obtained from the entangled state $\left|A_{j}\right\rangle$ by superposition with the singlet. The singlet, being orthogonal to all three states $\left|a_{j}\right\rangle$ of our initial ensemble, does not contribute to the probabilities of any of the six outcomes of our unentangled measurement. Moreover, the fact that the singlet has the same weight in all six of the POVM elements of this measurement ensures that its presence will have no effect on the mutual information. In other words, the effective equivalence between the measurements of Sections 2 and 3 follows fairly directly from Eq. (27).

In light of this observation, it is reasonable to ask whether we could have seen from the outset, without going through the construction in Section 3, that some superpositions of the form

$$
\beta\left|A_{j}\right\rangle-\gamma|S\rangle \text { and } \beta\left|A_{j}\right\rangle+\gamma|S\rangle
$$

would be separable for all $j=0,1,2$. If so, then we could have tried to use this fact to construct a suitable POVM.

In fact we could have seen this. A simple measure of the entanglement of a pure two-qubit state $|v\rangle$ is the concurrence $C$ [20], which can be written as

$$
C=\left|\left\langle\bar{v}\left|\sigma_{y} \otimes \sigma_{y}\right| v\right\rangle\right|,
$$

where $\langle\bar{v}|$ is obtained from $\langle v|$ by complex conjugation in the standard basis. For our present purpose it is useful to consider the related quantity

$$
c=\left\langle\bar{v}\left|\sigma_{y} \otimes \sigma_{y}\right| v\right\rangle
$$

which is $C$ without the absolute value. Evaluating $c$ for the superposition $\beta\left|A_{0}\right\rangle+\gamma|S\rangle$, we have

$$
\begin{aligned}
c & =\left(\beta \left\langleA_{0}|+\gamma\langle S|)\left(\sigma_{y} \otimes \sigma_{y}\right)\left(\beta\left|A_{0}\right\rangle+\gamma|S\rangle\right)\right.\right. \\
& =\beta^{2}\left\langle A_{0}\left|\sigma_{y} \otimes \sigma_{y}\right| A_{0}\right\rangle+\gamma^{2}\left\langle S\left|\sigma_{y} \otimes \sigma_{y}\right| S\right\rangle=\beta^{2} / 3-\gamma^{2},
\end{aligned}
$$


where we have used the fact that $\left\langle A_{0}\left|\sigma_{y} \otimes \sigma_{y}\right| S\right\rangle=0$, and in the last step we have evaluated $\left\langle A_{0}\left|\sigma_{y} \otimes \sigma_{y}\right| A_{0}\right\rangle$ starting from Eq. (23). From Eq. (31) it is clear that we can choose real $\beta$ and $\gamma$ so that $c=0$ for the state $\beta\left|A_{0}\right\rangle+\gamma|S\rangle$, and that the same $\beta$ and $\gamma$ will work for the superposition $\beta\left|A_{0}\right\rangle-\gamma|S\rangle$. So these states can both be made unentangled. The values of $\beta$ and $\gamma$ derived in this way are $\beta= \pm \sqrt{3} / 2$ and $\gamma= \pm 1 / 2$, in agreement with Eq. (24). The states given in Eq. (28) with $j=1$ and $j=2$ can similarly be made unentangled, with the same values of $\beta$ and $\gamma$, since the unitary operator $U$, which takes $\left|A_{j}\right\rangle$ to $\left|A_{j+1}\right\rangle$ and leaves $|S\rangle$ invariant, commutes with $\sigma_{y} \otimes \sigma_{y}$.

So far in this section we have focused on the requirement that the states in Eq. (28) be separable. But we also want these states to constitute a POVM. That is, we want

$$
\frac{2}{3} \sum_{j=0}^{2}\left[( \beta | A _ { j } \rangle + \gamma | S \rangle ) \left(\bar{\beta}\left\langle A_{j}|+\bar{\gamma}\langle S|)+\left(\beta\left|A_{j}\right\rangle-\gamma|S\rangle\right)\left(\bar{\beta}\left\langle A_{j}\right|-\bar{\gamma}\langle S|\right)\right]=I\right.\right.
$$

This requirement can be simplified to the form

$$
4|\gamma|^{2}|S\rangle\left\langle\left. S|+(4 / 3)| \beta\right|^{2} \sum_{j=0}^{2} \mid A_{j}\right\rangle\left\langle A_{j}\right|=I \text {. }
$$

Now, we know that

$$
|S\rangle\left\langle S\left|+\sum_{j=0}^{2}\right| A_{j}\right\rangle\left\langle A_{j}\right|=I
$$

So the six states given in Eq. (28) will constitute a POVM only if $|\beta|^{2}=3 / 4$ and $|\gamma|^{2}=1 / 4$. If we take $\beta$ and $\gamma$ to be real, we get the same values that we obtained above by requiring that the states in Eq. (28) be separable.

We thus have two distinct arguments leading to the conclusion that $\beta^{2}$ should be three times as large as $\gamma^{2}$ (assuming now that $\beta$ and $\gamma$ are real). The latter argument - based on the requirement that the states of Eq. (28) constitute a POVM - ultimately boils down to the fact that there are three $\left|A_{j}\right\rangle$ 's and only one $|S\rangle$. The former argument-based on the requirement that the states of Eq. (28) be separable - ultimately boils down to the fact that each of the $\left|A_{j}\right\rangle$ 's is only $1 / 3$ as entangled as the singlet state, entanglement being measured by the concurrence. If one could find some general principle ensuring that the sum of the concurrences of the $\left|A_{j}\right\rangle$ 's is equal 
to the concurrence of $|S\rangle$, then these two facts could be seen as closely related and the existence of our unentangled and (apparently) optimal POVM would be less surprising. However, I am not aware of any such general principle; so for the time being, I am inclined to think that there is something special about the double-trine ensemble that makes these two distinct arguments lead to the same conclusion and thereby allows the construction of an optimal measurement that is unentangled.

\section{Discussion}

This paper has focused on one particular ensemble of states, the doubletrine. When Asher Peres and I considered this case originally, our question was whether the three states of the ensemble could be distinguished better by a global measurement than by any sequence of local measurements. The evidence strongly suggests that a global measurement is indeed better. But among global measurements, one can consider two kinds: those whose POVM operators are all tensor products, and those that include at least one entangled operator. In the present paper I have asked whether, for the double-trine ensemble, an unentangled POVM can be as good as the best entangled measurement (at least the best that is known). We have seen that a specific unentangled POVM is indeed just as good, and we have found a couple of ways of constructing it. In this sense the double-trine ensemble does not exhibit the strongest kind of nonlocality that one can imagine for an ensemble of product states.

This result raises the following question which I mentioned in the Introduction. Can every ensemble of product states be distinguished just as well by an unentangled measurement as by an entangled measurement? In other words, if the states that one is trying to distinguish are products, is it sufficient to consider measurement outcomes that are also products? This question has apparently not been answered in the literature, though much progress has been made on questions of distinguishability by local measurements [21, 22, 23, 16, 24, 25, 26]. It is known that certain sets of orthogonal product states that cannot be distinguished by a sequence of local measurements can be distinguished by separable measurements [7]. We also know that for some ensembles of unentangled mixed states (which are not product states but can be expressed as mixtures of product states), there exists no unentangled measurement achieving the optimal mutual information [5, 27]. 
However, this fact does not provide an answer to our current question. The set of all product states is much smaller than the set of all unentangled states.

In the preceding section, we saw, for our specific ensemble, how one could start with an entangled measurement and use it to construct an unentangled measurement that provided just as much information. Our method was to superpose, with each of the states representing a possible outcome of the entangled measurement, a certain proportion of another state (the singlet) which was orthogonal to each element of the given ensemble. One can imagine trying to generalize this construction, but it is not at all clear from this one example how one might do this. In any case, it would be good to settle the question about the power of unentangled measurements. If it turns out that every ensemble of product states can be distinguished optimally by an unentangled measurement, then, in a sense, quantum mechanics is not as nonlocal a theory as one might have imagined. 


\section{References}

[1] M. Ban, K. Yamazaki, and O. Hirota, Phys. Rev. A 55, 22 (1997).

[2] D. Brody and B. Meister, Phys. Rev. Lett. 76, 1 (1996).

[3] A. Acin, E. Bagan, M. Baig, L. I. Masanes, and R. Muñoz-Tapia, Phys. Rev. A 71, 032338 (2005).

[4] A. Peres and W. K. Wootters, Phys. Rev. Lett. 66, 1119 (1991).

[5] C. H. Bennett, D. P. DiVincenzo, C. A. Fuchs, T. Mor, E. Rains, P. W. Shor, J. A. Smolin, and W. K. Wootters, Phys. Rev. A 59, 1070 (1999).

[6] C. H. Bennett, D. P. DiVincenzo, T. Mor, P. W. Shor, J. A. Smolin, and B. M. Terhal, Phys. Rev. Lett. 82, 5385 (1999).

[7] D. P. DiVincenzo, T. Mor, P. W. Shor, J. A. Smolin, and B. M. Terhal, Comm. Math. Phys. 238, 379 (2003).

[8] R. D. Gill and S. Massar, Phys. Rev. A 61, 042312 (2000).

[9] P. B. Slater, J. Phys. A 34, 7029 (2001).

[10] E. Bagan, M. A. Ballester, R. Muñoz-Tapia, and O. Romero-Isart, quant-ph/0505083.

[11] P. W. Shor, in Quantum Communication, Measurement, and Computing, Proceedings of the Fifth International Conference on Quantum Communication, Measurement, and Computing, Capri, 1998 (Kluwer Academic/Plenum Publishers, New York, 2001), p. 107; P. W. Shor, IBM J. Res. \& Dev. 48 (1), 115 (2004).

[12] M. Ban, K. Kurokawa, R. Momose, and O. Hirota, Int. J. Theor. Phys. 36, 1269 (1997).

[13] M. Sasaki, K. Kato, M. Izutsu, and O. Hirota, Phys. Rev. A 58, 146 (1998).

[14] Y. C. Eldar and G. D. Forney Jr., IEEE Trans. Inf. Theory 47, 858 (2001). 
[15] B. Groisman and L. Vaidman, J. Phys. A 34, 6881 (2001).

[16] J. Walgate and L. Hardy, Phys. Rev. Lett. 89, 147901 (2002).

[17] S. Massar and S. Popescu, Phys. Rev. Lett. 74, 1259 (1995).

[18] A. S. Holevo, Probl. Peredachi Inf. 9, 31 (1973).

[19] M. Sasaki, S. M. Barnett, R. Jozsa, M. Osaki, and O. Hirota, Phys. Rev. A 59, 3325 (1999).

[20] S. Hill and W. K. Wootters, Phys. Rev. Lett. 78, 5022 (1997); W. K. Wootters, Phys. Rev. Lett. 80, 2245 (1998).

[21] J. Walgate, A. J. Short, L. Hardy, and V. Vedral, Phys. Rev. Lett. 85, $4972(2000)$.

[22] S. Virmani, M. F. Sacchi, M. B. Plenio, and D. Markham, Phys. Lett. A 288, 62 (2001).

[23] S. Ghosh, G. Kar, A. Roy, A. Sen(De), and U. Sen, Phys Rev. Lett. 87, 277902 (2001); S. Ghosh, G. Kar, A. Roy, D. Sarkar, A. Sen(De), and U. Sen, Phys. Rev. A 65, 062307 (2002).

[24] P. X. Chen and C. Z. Li, Phys. Rev. A 68, 062107 (2003).

[25] B. M. Terhal, D. P. DiVincenzo, and D. W. Leung, Phys. Rev. Lett. 86, 5807 (2001).

[26] M. Horodecki, A. Sen(De), U. Sen, and K. Horodecki, Phys. Rev. Lett. 90, 047902 (2003).

[27] D. P. DiVincenzo, D. W. Leung, and B. M. Terhal, IEEE Trans. Inf. Theory 48, 580 (2002). 